\title{
Adrenal Gland Composite Pheochromocytoma
}

National Cancer Institute

\section{Source}

National Cancer Institute. Adrenal Gland Composite Pheochromocytoma. NCl

Thesaurus. Code C48306.

A benign or malignant neoplasm arising from the adrenal medulla. It combines

morphologic characteristics of pheochromocytoma and neuroectodermal tumors such as neuroblastoma, ganglioneuroma, ganglioneuroblastoma, or peripheral nerve sheath tumor. 